\title{
An in vitro evaluation of guanfacine as a substrate for P-glycoprotein
}

This article was published in the following Dove Press journal:

Neuropsychiatric Disease and Treatment

25 August 20II

Number of times this article has been viewed

\author{
Nancy K Gillis' \\ Hao-Jie Zhu',2 \\ John S Markowitz ${ }^{1,2}$ \\ 'Department of Pharmacotherapy \\ and Translational Research, ${ }^{2}$ Center \\ for Pharmacogenomics, University \\ of Florida, Gainesville, FL, USA
}

Correspondence: John S Markowitz University of Florida College of Pharmacy, Department of Pharmacotherapy and Translational Research, I600 SW Archer Road, Room PG-23, Gainesville,

FL 326I0-0486, USA

Tel +I 3522736262

Fax + I 3522736121

Email jmarkowitz@cop.ufl.edu
Background: With a US Food and Drug Administration-labeled indication to treat attention-deficit/hyperactivity disorder (ADHD), the nonstimulant guanfacine has become the preferred $\alpha_{2}$-agonist for ADHD treatment. However, significant interindividual variability has been observed in response to guanfacine. Consequently, hypotheses of a contributing interaction with the ubiquitously expressed drug transporter, P-glycoprotein (P-gp), have arisen. We performed an in vitro study to determine if guanfacine is indeed a substrate of P-gp.

Methods: Intracellular accumulation of guanfacine was compared between P-gp expressing LLC-PK1/MDR1 cells and P-gp-negative LLC-PK1 cells to evaluate the potential interaction between P-gp and guanfacine. Cellular retention of guanfacine was analyzed using a high-performance liquid chromatographic-ultraviolet method. Rhodamine6G, a known P-gp substrate, was included in the study as a positive control.

Results: At guanfacine concentrations of $50 \mu \mathrm{M}$ and $5 \mu \mathrm{M}$, intracellular accumulation of guanfacine in LLC-PK1/MDR1 cells was, $35.9 \% \pm 4.8 \%$ and $49.0 \% \pm 28.3 \%$ respectively, of that in LLC-PK1 cells. In comparison, the concentration of rhodamine6G, the positive P-gp substrate, in LLC-PK1/MDR1 cells was only 5\% of that in LLC-PK1 cells.

Conclusion: The results of the intracellular accumulation study suggest that guanfacine is, at best, a weak P-gp substrate. Therefore, it is unlikely that P-gp, or any genetic variants thereof, are a determining factor in the interindividual variability of response observed with guanfacine therapy.

Keywords: guanfacine, P-glycoprotein, substrate, intracellular uptake

\section{Introduction}

Guanfacine, initially marketed as an antihypertensive agent, is a selective $\alpha_{2}$-adrenergic agonist believed to exert its antihypertensive activity by decreasing sympathetic tone in the central nervous system and reducing vascular resistance. ${ }^{1}$ However, because of its preferential effects on $\alpha_{2 \mathrm{~A}}$-adrenoreceptors, which are believed to play a role in attentional and organizational functions in the prefrontal cortex, guanfacine became a drug of interest in the treatment of attentiondeficit/hyperactivity disorder (ADHD). ${ }^{2,3}$ Although psychostimulants, such as methylphenidate and amphetamine, are considered first-line treatments for ADHD, they are discontinued in approximately $5 \%$ of patients due to lack of efficacy or unwanted side effects. ${ }^{4}$ When psychostimulants are ineffective or only partially effective in reducing target symptoms, clinicians may opt to utilize nonstimulant medications, such as the $\alpha_{2}$-agonists clonidine and guanfacine, as either adjunctive treatments or monotherapy. Because of its greater selectivity for 
the $\alpha_{2 \mathrm{~A}}$-adrenoreceptor, a longer half-life, a potentially decreased side effect burden, and a US Food and Drug Administration-labeled indication to treat ADHD as monotherapy or as an adjunct to stimulant medications, guanfacine has become the preferred $\alpha_{2}$-agonist for use in ADHD. ${ }^{5}$ Additionally, a number of published studies have demonstrated the efficacy of guanfacine in reducing target symptoms of ADHD in children and adolescents. ${ }^{5-7}$

As is the case with the more traditional psychostimulants, methylphenidate and amphetamine, significant interindividual variability in response to guanfacine has been reported. For example, in a prospective trial of guanfacine in children with comorbid pervasive developmental disorder and ADHD, the improvement from baseline ADHD symptom scores ranged widely (ie, -1 to 41 points). ${ }^{8}$ Similarly, in a large retrospective chart review of children with pervasive developmental disorders receiving guanfacine, the rate of positive response ranged from $13 \%$ to $39 \%$, depending on the patient population assessed. ${ }^{9}$ A trend indicating clear superiority of guanfacine over placebo, albeit with significant interindividual variability in endpoint ratings of ADHD symptoms, is a consistent finding in the published literature. ${ }^{6,10}$

Interindividual variation in drug response and disposition may be attributed to varied drug transporter activity, which is mediated by an array of single nucleotide polymorphisms (SNPs), as well as other endogenous and exogenous substances that may serve as transporter inhibitors or inducers. ${ }^{11,12} \mathrm{P}$-glycoprotein (P-gp) is the most thoroughly studied member of the adenosine triphosphate-binding cassette transporter superfamily, and is expressed throughout the body in the intestinal epithelium, hepatocytes, renal tubular cells, adrenal gland, blood-brain barrier, bloodtestis barrier, and blood-placenta barrier. ${ }^{13}$ In humans, P-gp is encoded by the $A B C B 1 / M D R 1$ gene. ${ }^{14,15}$ Because of its ubiquitous expression and broad specificity, changes in P-gp expression or efflux activity induced by drug treatments, diet, environmental factors, or SNPs can greatly impact drug disposition, pharmacokinetics, and clinical response. ${ }^{16,17}$ Hence, identification of $\mathrm{P}$-gp substrates is important for therapeutic optimization and the avoidance of drug-drug interactions.

Several published studies have investigated the potential interaction between guanfacine and P-gp. Mahar Doan et al conducted an in vitro study to determine whether guanfacine is a substrate of P-gp utilizing a Transwell cell culture model with P-gp-expressing Madin Darby canine kidney cells. The ratio of the transport velocity of guanfacine in the direction of basolateral-to-apical to apical-to-basolateral was found to be 1.2 , indicating that guanfacine is not a P-gp substrate. ${ }^{18}$ Similarly, based upon a computational method (topological substructural molecular design approach), other investigators predicted that guanfacine would be unlikely to serve as a P-gp substrate. ${ }^{19}$

Although these data suggest that guanfacine is not a P-gp substrate, a recently published clinical study examined the potential influence of the known SNPs variant of P-gp, $\mathrm{C} 3435 \mathrm{~T}$, on response to guanfacine treatment in children diagnosed with ADHD. The authors concluded that patients genotyped and found to carry the C3435T variant showed significantly less clinical improvement in ADHD symptoms relative to patients who were wild-type for the gene. ${ }^{20}$ The $\mathrm{C} 3435 \mathrm{~T}$ variant has previously been shown to influence both gene expression and substrate specificity of P-gp. ${ }^{17,21}$ Thus, the association between therapeutic response and the C3435T SNPs suggests that P-gp plays a role in the transport of guanfacine. Therefore, the purpose of this study was to use a different cell line (human P-gp-overexpressing porcine kidney epithelial cell line LLC-PK1/MDR1) and a different in vitro model (intracellular uptake) relative to that previously published to determine if guanfacine is indeed a P-gp substrate.

\section{Materials and methods Materials}

The porcine kidney epithelial cell line LLC-PK1 and human P-gp-overexpressing mutant LLC-PK1/MDR1 cells were kindly provided by Dr Kari Kivistö (Dr Margarete Fischer-Bosch Institute of Clinical Pharmacology, Stuttgart, Germany). Fetal bovine serum, trypsin, and Dulbecco's Modified Eagle Medium containing $4500 \mathrm{mg} / \mathrm{L}$ glucose, $4 \mathrm{mM}$ L-glutamine, and sodium pyruvate were obtained from Hyclone Co (Logan, UT). Dulbecco's phosphate-buffered saline, penicillin, and streptomycin were purchased from Mediatech Inc (Herndon, VA). Guanfacine and rhodamine6G were obtained from Sigma Co (St Louis, MO). The Pierce bicinchoninic acid protein assay kit (Thermo Scientific, Rockford, IL) was used to determine protein concentrations. All other agents were of high analytical grade and commercially available.

\section{Cell cultures and intracellular uptake studies}

LLC-PK1 and LLC-PK1/MDR1 cells were cultured at $37^{\circ} \mathrm{C}$ in Dulbecco's Modified Eagle Medium supplemented with $10 \%$ fetal bovine serum, $1 \%$ penicillin, and streptomycin 
in an atmosphere of $5 \% \mathrm{CO}_{2}$ and $95 \%$ relative humidity. Cells $(1 \mathrm{~mL})$ were seeded into $24-w e l l$ plates at a density of $1 \times 10^{5}$ cells $/ \mathrm{mL}$. Culture medium was replaced every two days until cells reached confluence. To investigate the potential influence of P-gp on guanfacine transport, intracellular accumulation of guanfacine was measured in LLC-PK1/MDR1 cells and P-gp-negative LLC-PK1 cells. Rhodamine6G, a well-known P-gp substrate, was included as a positive control. After cells reached confluence, the culture medium was replaced by transport buffer (serum-free Dulbecco's Modified Eagle Medium) for a 30-minute preincubation at $37^{\circ} \mathrm{C}$. Transport buffer was then removed and the test compounds $(5 \mu \mathrm{M}$ rhodamine $6 \mathrm{G}, 5 \mu \mathrm{M}$ guanfacine, or $50 \mu \mathrm{M}$ guanfacine) were added, and an additional 60-minute incubation period commenced. Next, the solutions were discarded, and the cells were washed three times with ice-cold Dulbecco's phosphate-buffered saline and solubilized with $1 \%$ Triton X-100. Intracellular accumulation of rhodamine $6 \mathrm{G}$ was measured using a fluorescent microplate reader with a $530 \mathrm{~nm}$ excitation and a $580 \mathrm{~nm}$ emission wavelength, which is a well established method. ${ }^{22}$ Cellular retention of guanfacine was analyzed by the high-performance liquid chromatographic assay described below. The concentrations of rhodamine $6 \mathrm{G}$ and guanfacine were calculated from their respective standard curves, and standardized with the cellular protein content determined by a Pierce bicinchoninic acid protein kit.

\section{High-performance liquid chromatographic analysis}

A novel high-performance liquid chromatographic-ultraviolet detection method for guanfacine quantification was developed for this study. Briefly, $100 \mu \mathrm{L}$ of acetonitrile containing the internal standard, p-nitrophenol $100 \mu \mathrm{M}$, was added to a $100 \mu \mathrm{L}$ sample and vortexed. Samples were centrifuged at $13,200 \mathrm{rpm}$ at $4^{\circ} \mathrm{C}$ for 30 minutes to remove proteins. The supernatant $(50 \mu \mathrm{L})$ was injected into the high-performance liquid chromatography column for analysis. Separation was performed on a reverse-phase column (Luna $5 \mu \mathrm{C} 8$, $250 \times 4.6 \mathrm{~mm}$ ) at $40^{\circ} \mathrm{C}$. The mobile phase consisted of $25 \%$ acetonitrile and $75 \% \mathrm{KH}_{2} \mathrm{PO}_{4}(10 \mathrm{mM}, \mathrm{pH} 4)$, and the flow rate was $1.0 \mathrm{~mL} / \mathrm{min}$. Guanfacine was detected by ultraviolet absorption at $220 \mathrm{~nm}$.

\section{Data analysis}

All data are presented as means \pm standard deviation. Intracellular concentrations of rhodamine $6 \mathrm{G}$ and guanfacine in LLC-PK1/MDR1 cells are expressed as the percentages of that in LLC-PK1 cells. A two-tailed unpaired Student's $t$-test was used for data analyses, with a $P$ value of $\leq 0.5$ considered statistically significant.

\section{Results}

The P-gp substrate properties of guanfacine were assessed by measuring intracellular drug accumulations in LLCPK1 and LLC-PK1/MDR1 cells. The results shown in Figure 1 indicate that, at a guanfacine concentration of $50 \mu \mathrm{M}$, the intracellular concentration of guanfacine was three-fold higher in LLC-PK1 cells than in LLC-PK1/ MDR1 cells $(35.9 \% \pm 4.8 \%$ of LLC-PK1 cell uptake, $P<0.01)$. Similarly, at a guanfacine concentration of $5 \mu \mathrm{M}$, the intracellular concentration of guanfacine was two-fold higher in LLC-PK1 cells than in LLC-PK1/MDR1 cells $(49.0 \% \pm 28.3 \%, P<0.05)$. By comparison, the concentration of the positive P-gp control substrate, rhodamine6G, in LLC-PK1/MDR1 cells was only $5 \%$ of that in LLCPK1 cells. Overall, these results suggest that guanfacine is, at best, a weak substrate for P-gp.

\section{Discussion}

An estimated 100,000 patients die each year of adverse drug reactions in the US, thus making adverse drug reactions one of the leading causes of death in hospitalized patients. ${ }^{23}$ Some of these deaths are caused by drug-drug interactions. Most drug interactions occur as an unexpected result of changes in pharmacokinetics due to drug coadministration. Combined pharmacotherapy in child, adolescent, and adult psychiatric patients has recently become a growing practice. ${ }^{24-26}$ Because ADHD therapeutic agents are the most commonly used psychotropic drugs in children

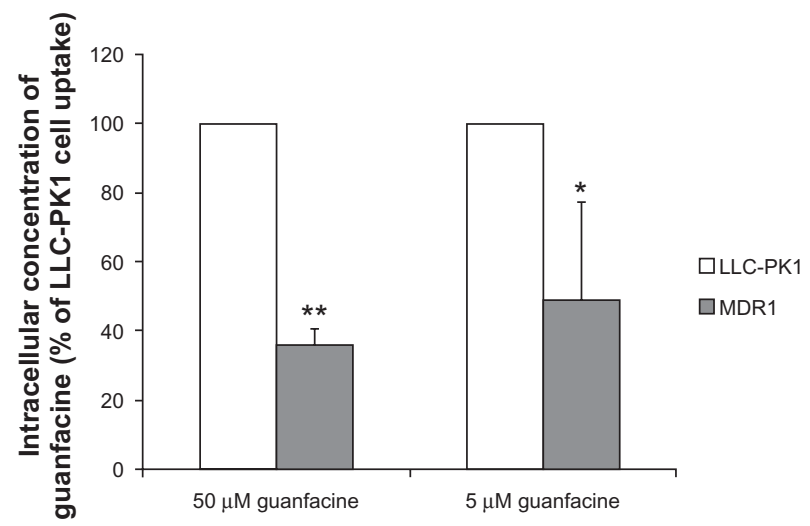

Figure I Intracellular concentrations of guanfacine in LLC-PKI and LLC-PKI/ MDRI cells. Each column represents the mean percentages of intracellular guanfacine concentrations relative to that in LLC-PKI cells, with a bar representing the standard deviation of three independent measurements.

Notes: $* P<0.05$; ** $P<0.01$. 
and adolescents and are often used in combination with other therapeutic agents on an acute or chronic basis, the potential for drug-drug interactions, at least in theory, is extremely high. ${ }^{27-29}$

Interaction of psychotropic drugs with P-gp has been well documented in recent years. For example, Uhr et al demonstrated that a series of antidepressant medications, including venlafaxine and paroxetine, are substrates for P-gp. ${ }^{30}$ Additionally, Wang et al reported that the antipsychotic risperidone and its active metabolite, 9-hydroxy-risperidone, are both P-gp substrates. ${ }^{31}$ Other psychotropic agents that have been documented as substrates of P-gp to some degree include $(R)$-methadone, $(S)$-methadone, olanzapine, $d$-modafinil, $l$-modafinil, and $d$-methylphenidate. ${ }^{29,32,33}$ Because P-gp is the most important efflux transporter at the blood-brain barrier, entry of these substrates into the central nervous system is greatly limited. Furthermore, a number of psychotropic drugs have been found to be P-gp inhibitors, which may affect disposition of coadministered P-gp substrates. ${ }^{29,34,35}$

Prominent variability of response has been reported in patients taking guanfacine to treat ADHD. ${ }^{6,8-10}$ In a recent study, variation in response to guanfacine correlated to a single nucleotide polymorphism of the $M D R l$ gene, the gene encoding for human P-gp. ${ }^{20}$ The present study was therefore performed to determine if guanfacine, like many other psychotropic drugs, is a $\mathrm{P}$-gp substrate.

The results of the intracellular accumulation study of guanfacine in P-gp-expressing cells suggest that guanfacine is, at best, a weak P-gp substrate. Specifically, the accumulation of guanfacine in LLC-PK1/MDR1 cells was approximately $30 \%-50 \%$ of that in LLC-PK1 cells under our experimental conditions. In comparison, the intracellular concentration of the established P-gp substrate rhodamine6G in LLC-PK1/MDR1 cells was only 5\% of that in control cells. Thus, it appears the role of P-gp in guanfacine transport is minor, and P-gp is unlikely to be one of the determinants of the interindividual variability in response to guanfacine therapy. Thus, the reported effect of the MDR1 SNP C3435T on the response to guanfacine treatment must be interpreted carefully and warrants further investigation.

\section{Acknowledgment}

We would like to acknowledge the University of Florida Department of Pharmacotherapy and Translational Research Summer Research Training Program for providing support to perform this research.

\section{Disclosure}

The authors report no conflicts of interest in this work.

\section{References}

1. Sorkin EM, Heel RC. Guanfacine: A review of its pharmacodynamic and pharmacokinetic properties, and therapeutic efficacy in the treatment of hypertension. Drugs. 1986;31(4):301-336.

2. Arnsten AF, Cai JX, Goldman-Rakic PS. The alpha-2 adrenergic agonist guanfacine improves memory in aged monkeys without sedative or hypotensive side effects: Evidence for alpha-2 receptor subtypes. J Neurosci. 1988;8(11):4287-4298.

3. Avery R, Franowicz JCS, Studholme C, van Dyck CH, Arnsten AFT. The alpha-2A-adrenoceptor agonist, guanfacine, increases regional cerebral blood flow in dorsolateral prefrontal cortex and improves accuracy in monkeys performing a spatial working memory task. Neuropsychopharmacology. 2000;23(3):240-249.

4. Crenshaw TM, Kavale KA, Forness SR, Reeve RE. Attention deficit hyperactivity disorder and the efficacy of stimulant medication: A meta-analysis. In: Scruggs TE, Mastropieri MA, editors. Advances in Learning and Behavioral Disabilities. Greenwich, CT: JAI Press; 1999.

5. Biederman J, Melmed RD, Patel A, et al; for SPD503 Study Group. A randomized, double-blind, placebo-controlled study of guanfacine extended release in children and adolescents with attention-deficit/ hyperactivity disorder. Pediatrics. 2008;121(1):e73-e84.

6. Newcorn JH, Schulz KP, Halperin JM. Adrenergic agonists: Clonidine and guanfacine. In: Martin A, Scahill L, Charney DS, Leckman JF, editors. Pediatric Psychopharmacology: Principles and Practice. New York, NY: Oxford; 2003.

7. Scahill L, Chappel PB, Kim YS, et al. A placebo-controlled study of guanfacine in the treatment of children with tic disorders and attention deficit hyperactivity disorder. Am J Psychiatry. 2001;158(7):1067-1074.

8. Scahill L, Aman MG, McDougle CJ, et al. A prospective open trial of guanfacine in children with pervasive developmental disorders. $J$ Child Adolesc Psychopharmacol. 2006;16(5):589-598.

9. Posey DJ, Puntney JI, Sasher TM, Kem DL, McDougle CJ. Guanfacine treatment of hyperactivity and inattention in pervasive developmental disorders: A retrospective analysis of 80 cases. J Child Adolesc Psychopharmacol. 2004;14(2):233-241.

10. Cummings DD, Singer HS, Krieger M, Miller TL, Mahone EM. Neuropsychiatric effects of guanfacine in children with mild Tourette syndrome: A pilot study. Clin Neuropharmacol. 2002;25(6): 325-332.

11. Beringer PM, Slaughter RL. Transporters and their impact on drug disposition. Ann Pharmacother. 2005;39(6):1097-1108.

12. Bosch TM, Meijerman I, Beijnen JH, Schellens JH. Genetic polymorphisms of drug-metabolising enzymes and drug transporters in the chemotherapeutic treatment of cancer. Clin Pharmacokinet. 2006;45(3):253-285.

13. Thiebaut F, Tsuruo T, Hamada H, Gottesman MM, Pastan I, Willingham MC. Cellular localization of the multidrug-resistance gene product P-glycoprotein in normal human tissues. Proc Natl Acad Sci U S A. 1987;84(21):7735-7738.

14. Schuetz EG, Beck WT, Schuetz JD. Modulators and substrates of P-glycoprotein and cytochrome P4503A coordinately up-regulate these proteins in human colon carcinoma cells. Mol Pharmacol. 1996; 49(2):311-318.

15. Wacher VJ, Wu CY, Benet LZ. Overlapping substrate specificities and tissue distribution of cytochrome P4503A and P-glycoprotein: Implications for drug delivery and activity in cancer chemotherapy. Mol Carcinog. 2000;13(3):129-134.

16. Hitzl M, Drescher S, van der Kuip H, et al. The C3435T mutation in the human MDR1 gene is associated with altered efflux of the P-glycoprotein substrate rhodamine 123 from CD56+ natural killer cells. Pharmacogenetics. 2001;11(4):293-298. 
17. Hoffmeyer S, Burk O, von Richter O, et al. Functional polymorphisms of the human multidrug-resistance gene: Multiple sequence variations and correlation of one allele with P-glycoprotein expression and activity in vivo. Proc Natl Acad Sci U S A. 2000;97(7):3473-3478.

18. Mahar Doan KM, Humphreys JE, Webster LO, et al. Passive permeability and P-glycoprotein-mediated efflux differentiate central nervous system (CNS) and non-CNS marketed drugs. J Pharmacol Exp Ther. 2002;303(3):1029-1017.

19. Cabrera MA, González I, Fernández C, Navarro C, Bermejo M. A topological substructural approach for the prediction of P-glycoprotein substrates. J Pharm Sci. 2006;95(3):589-606.

20. McCracken JT, Aman MG, McDougle CJ, et al. Possible influence of variant of the $\mathrm{P}$-glycoprotein gene $(M D R 1 / A B C B 1)$ on clinical response to guanfacine in children with pervasive developmental disorders and hyperactivity. J Child Adolesc Psychopharmacol. 2010;20(1):1-5.

21. Kimchi-Sarfaty C, Oh JM, Kim I-W, et al. A "silent" polymorphism in the MDRl gene changes substrate specificity. Science. 2007; 315(5811):525-528.

22. Sarver JG, Klis WA, Byers JP, Erhardt PW. Microplate screening of the differential effects of test agents on Hoechst 33342, rhodamine 123, and rhodamine $6 \mathrm{G}$ accumulation in breast cancer cells that overexpress P-glycoprotein. J Biomol Screen. 2002;7(1):29-34.

23. Lazarou J, Pomeranz BH, Corey PN. Incidence of adverse drug reactions in hospitalized patients: A meta-analysis of prospective studies. JAMA 1998;279(15):1200-1205.

24. Connor DF, Ozbayrak KR, Kusiak KA, Caponi AB, Melloni RH Jr. Combined pharmacotherapy in children and adolescents in a residential treatment center. J Am Acad Child Adolesc Psychiatry. 1997;36(2): $248-254$

25. Safer DJ, Zito JM, DosReis S. Concomitant psychotropic medication for youths. Am J Psychiatry. 2003;160(3):438-449.
26. Wilens TE, Spencer T, Biederman J, Wozniak J, Connor D. Combined pharmacotherapy: An emerging trend in pediatric psychopharmacology. J Am Acad Child Adolesc Psychiatry. 1995;34(1):110-112.

27. Greenhill LL, Halperin JM, Abikoff H. Stimulant medications. J Am Acad Child Adolesc Psychiatry. 1999;38(3):503-512.

28. Spencer T, Biederman J, Wilens T. Attention-deficit/hyperactivity disorder and comorbidity. Pediatr Clin North Am. 1999;46(5):915-927.

29. Zhu H-J, Wang J-S, Donovan JL, et al. Interactions of attention-deficit/ hyperactivity disorder therapeutic agents with the efflux transporter P-glycoprotein. Eur J Pharm Sci. 2008;578(2-3):148-158.

30. Uhr M, Grauer MT, Holsboer F. Differential enhancement of antidepressant penetration into the brain in mice with abcblab (mdr1ab) P-glycoprotein gene disruption. Biol Psychiatry. 2003;54(8):840-846.

31. Wang J-S, Ruan Y, Taylor RM, Donovan JL, Markowitz JS, DeVane CL. The brain entry of risperidone and 9-hydroxyrisperidone is greatly limited by P-glycoprotein. J Neuropsychopharmacology. 2004;7(4):415-419.

32. Wang J-S, Ruan Y, Taylor RM, Donovan JL, Markowitz JS, DeVane CL. Brain penetration of methadone $(R)$ - and $(S)$-enantiomers is greatly increased by P-glycoprotein deficiency in the blood-brain barrier of abcbla gene knockout mice. Psychopharmacology. 2004;173(1-2): $132-138$.

33. Wang J-S, Taylor R, Ruan Y, Donovan JL, Markowitz JS, DeVane CL. Olanzapine penetration into brain is greater in transgenic abcbla P-glycoprotein-deficient mice than FVB1 (wild-type) animals. J Neuropsychopharmacology. 2004;29(3):551-557.

34. Wang J-S, Zhu H-J, Markowitz JS, Donovan JL, DeVane CL. Evaluation of antipsychotic drugs as inhibitors of multidrug resistance transporter P-glycoprotein. Psychopharmacology. 2006;187(4):415-423.

35. Zhu H-J, Wang J-S, Markowitz JS, Donovan JL, Gibson BB, DeVane CL. Risperidone and paliperidone inhibit P-glycoprotein activity in vitro. Neuropsychopharmacology. 2007;32(4):757-764.
Neuropsychiatric Disease and Treatment

\section{Publish your work in this journal}

Neuropsychiatric Disease and Treatment is an international, peerreviewed journal of clinical therapeutics and pharmacology focusing on concise rapid reporting of clinical or pre-clinical studies on a range of neuropsychiatric and neurological disorders. This journal is indexed on PubMed Central, the 'PsycINFO' database and CAS, and is the official

\section{Dovepress}

journal of The International Neuropsychiatric Association (INA). The manuscript management system is completely online and includes a very quick and fair peer-review system, which is all easy to use. Visit http://www.dovepress.com/testimonials.php to read real quotes from published authors. 